\title{
Utilisation des grains de polyamide pour estimer l'excrétion fécale de bovins sur pâturage sahélien
}

\author{
FC Mahler ${ }^{1}$, E Schlecht 2, M Sangaré ${ }^{3}, K^{2}$ Becker ${ }^{4}$ \\ I/CRISAT, BP 12404 Niamey, Niger ; 2/LRI, BP 12404 Niamey, Niger ; 3SRZ/S, BP 12 Niono, Mali; \\ 4 Inst Prod Anim Pays Trop et Subtrop, Univ Hohenheim, 70593 Stuttgart, Germany
}

Des grains de polyamide (PA, diamètre 2 à $3 \mathrm{~mm}$, densité 1,12 à $1,15 \mathrm{~g} / \mathrm{cm}^{3}$ ) sont utilisés comme marqueur externe pour estimer l'excrétion fécale, exprimée en matière sèche (MSF), de 8 à 12 boeufs zébus. Des expériences ont été menées à l'étable ( 7 essais) ainsi que sur pâturage naturel (5 essais). Dans les deux cas la ration était composée de fourrage vert ou sec, provenant du même pâturage naturel. Pour la moitié des animaux la ration était supplémentée avec des sous-produits agricoles. Des capsules en gélatine contenant 35 ou $45 \mathrm{~g}$ PA étaient administrées par voie orale chaque 12 heures, la dose étant ajustée à l'excrétion attendue à raison de $35 \mathrm{~g} \mathrm{PA} / \mathrm{kg}$ MSF. Le marqueur est récupéré sept jours durant par collection fécale totale et par prélèvement d'échantillons fécaux au rectum chaque 7 heures ("grab sampling"). Les échantillons sont séchés, moulus et tamisés $(1 \mathrm{~mm})$ pour récupérer les grains de PA. Le taux de récupération du marqueur est calculé. L'excrétion fécale est estimée sur la base de la concentration moyenne du marqueur dans les échantillons et du taux de récupération. Le résultat est comparé à l'excrétion totale mesurée.

Après ajustement, une période d'adaptation au marqueur de 4 jours à été fixée. A l'exception des rations dont la teneur en azote est inférieure à $8 \mathrm{~g} / \mathrm{kg}$ matière organique, le taux de récupération du marqueur PA est de $98,1 \%( \pm 7,4)$, indépendamment des qualité et quantité de la ration ingérée. La quantité de MSF estimée est bien corrélée avec l'excrétion mesurée $(r=0,98, n=62)$. Le taux de récupération du marqueur varie avec l'heure de prélèvement d'un échantillon sans qu'il y ait de relation statistique entre les deux, ni entre le taux de récupération de marqueur et la taille de l'échantillon rectal. En conséquence, l'estimation de l'excrétion n'est fiable que si l'échantillonnage couvre les 24 heures. Cependant, l'estimation reste acceptable lorsque l'on considère le sousensemble des échantillons diurnes ou le sousensemble des échantillons nocturnes ( $r=0,95, n=30$ dans les deux cas). L'application de ces derniers résultats requiert une validation pour une conduite du troupeau différente.

En conclusion, l'utilisation du marqueur PA est une méthode simple pour estimer l'excrétion fécale avec une précision comparable à d'autres marqueurs d'utilisation plus délicate comme l'oxyde de chrome ou l'ytterbium. La méthode est adaptée aux estimations d'excrétion fécale et d'ingestion fourragère de bovins en élevage extensif dans les environnements difficiles. 